\title{
Application of Gray B6 P Neural Network in Henan Coal Demand Forecast
}

\author{
Guohua GOU ${ }^{1, a}$ \\ ${ }^{1}$ Department of Modern Management, Zhengzhou Technical College, Zhengzhou 450121, China
}

\begin{abstract}
This paper studied the coal demand in the prediction accuracy problems. The traditional gray $\mathrm{GM}(1,1)$ model has the theoretical prediction problem of poor accuracy which leaded to less accurate prediction. A modified gray BP Neural Network forecasting model was used to predict the residual correction. The total consumption of coal as a major factor in variables was selected to construct forecast of coal demand The simulation results show that the proposed algorithm has better prediction accuracy and is an effective demand forecasting algorithm
\end{abstract}

\section{Introduction}

As the basic energy in Henan Province, coal accounts for nearly three-fourths of energy consumption and plays an important role in economic and social development. Scientific prediction of coal demand can overcome or eliminate the phenomenon of overproduction of coal and ensure the economy Development needs. Common prediction methods include neural network prediction method and gray system model prediction method. As the method used and the basic data taken are different, resulting in large differences in the prediction results, and the prediction accuracy is low.

This paper attempts to apply the gray BP neural network model to the coal demand forecasting field, and uses the model to predict the coal demand from the advantages of time series and nonlinear forecasting. Gray prediction model has the characteristics of less required sample data, no need to consider its distribution and trend, simple modeling and convenient operation, but it lacks self-learning, self-organizing and selfadaptive ability, and its ability of processing non-linear information is weak. The gray prediction model is used alone to predict the system with nonlinear relationship. The prediction result and the actual value will have larger errors and the prediction accuracy will not meet the requirements. Artificial neural network is an effective nonlinear modeling method, of which the error back propagation (BP) algorithm is currently more mature and widely used algorithm. BP neural network has a high degree of mapping ability and can approach any nonlinear function with arbitrary precision, which is more suitable for modeling some complex problems. At present, some scholars have combined the gray prediction model and neural network and applied it in many fields, and obtained the ideal prediction result.

Please submit sources files directly to the conference organizer. If the conference editors chose to provide print-ready PDF documents to the publisher, you have to submit high-resolution PDF file with all fonts embedded (see PDF guidelines) instead of the sources files. In this case, please remember that no final corrections will be made by the publisher.

\section{Establishment of Grey Neural Network Model}

\subsection{Gray theory prediction model}

The gray system theory can extract the valuable information by the generation and development of some known information, and realize the correct understanding and effective control of the system behavior. Gray model is established on the premise that the original data sequence is a smooth discrete function, the so-called smooth discrete function, in fact, requires the original data sequence is deterministic, that is a certain trend, the modeling steps of $\operatorname{GM}(1,1)$ are as follows:

Assume that time series have $\mathrm{n}$ observations, $\quad X^{(0)}=\left(x^{(0)}(1), x^{(0)}(2), \cdots, x^{(0)}(n)\right) \quad$ in which $x^{(0)}(k) \geq 0, k=1,2, \cdots, n$.

Generate new series by accumulating, $\quad X^{(1)}=\left(x^{(1)}(1), x^{(1)}(2), \cdots, x^{(1)}(n)\right) \quad$ in which $x^{(1)}(k)=\sum_{\mathrm{i}=1}^{\mathrm{k}} x^{(0)}(i), k=1,2, \cdots n . Z^{(1)} \quad$ is the immediate mean of $X^{(1)}$ 's sequence, $Z^{(1)}=\left(z^{(1)}(1), z^{(1)}(2), \cdots, z^{(1)}(n)\right) \quad$ in which

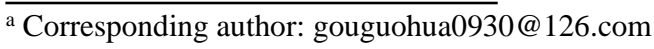




$$
z^{(1)}(k)=\frac{1}{2}\left[x^{(1)}(k)+x^{(1)}(k-1)\right], k=2,3, \cdots, n \text {.Th }
$$

en the corresponding differential equations of $\operatorname{GM}(1,1)$ model is:

$$
\begin{gathered}
x^{(0)}(k)+a z^{(1)}(k)=b \\
\text { If } \hat{a}=[a, b]^{\mathrm{T}} \text { is a constant column, and } \\
\boldsymbol{B}=\left[\begin{array}{cc}
-z^{(1)}(2) & 1 \\
-z^{(1)}(3) & 1 \\
\vdots & \vdots \\
-z^{(1)}(n) & 1
\end{array}\right], \quad \boldsymbol{Y}=\left[\begin{array}{c}
x^{(0)}(2) \\
x^{(0)}(3) \\
\vdots \\
x^{(0)}(n)
\end{array}\right]
\end{gathered}
$$

The least squares estimation parameter column of $\mathrm{GM}(1,1)$ model is satisfied

$$
\hat{a}=\left(B^{\mathrm{T}} B\right)^{-1} B^{\mathrm{T}} Y
$$

According to (1) the establishment of albino differential equation

$$
\frac{d x^{(1)}}{d t}+a x^{(1)}=b
$$

Solve the differential equations, then we can obtain

$$
x^{(1)}(t)=\left[x^{(0)}(1)-\frac{b}{a}\right] e^{-a t}+\frac{b}{a}
$$

The prediction formula of GM $(1,1)$ model is:

$$
\hat{x}^{(1)}(k+1)=\left[x^{(0)}(1)-\frac{b}{a}\right] e^{-a k}+\frac{b}{a} k=1,2, \cdots, n
$$

At this point you can use a cumulative, the result is:

$$
\begin{array}{r}
\hat{x}^{(0)}(k+1)=\hat{x}^{(1)}(k+1)-\hat{x}^{(1)}(k)=\left(1-e^{a}\right)\left(x^{(0)}(1)-\frac{u}{a}\right) e^{-a k} \\
k=1,2, \cdots, n \quad(6)
\end{array}
$$

In order to judge the merits and demerits of the gray model, we should also carry on the model precision test, the test method generally uses the residual, the posterior difference and so on the method, if passes the examination, then may use the model to carry on the forecast, otherwise should carry on the residual correction, to achieve the accuracy of the forecast.

\subsection{BP neural network prediction model}

BP neural network is the most widely used neural network model and algorithm. It is a model of information forward propagation and error back propagation. It is a feed forward network composed of nonlinear transformation units. The typical structure is shown in Figure 1.In the BP network, the input information is forwarded to the hidden layer node, the activation function (usually sigmoid function), then the hidden node information to the output layer nodes, the final output structure. If the desired result is not obtained at the output node, it is transferred backwards, the error information is returned along the original connection path, and the error is minimized by modifying the neuron weight of each layer.

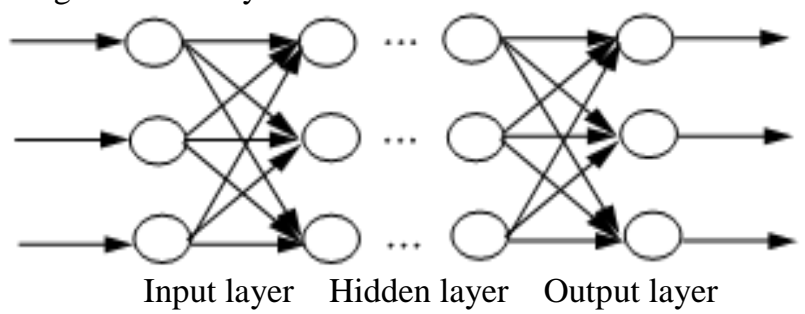

Fig. 1: BP neural network

For the three-layer BP network of Figure 1, the learning algorithm is as follows:

Step 1: Assign the connection weight ${ }^{W_{i j}}$ randomly and set the initial value for the threshold $\theta_{j}\left(W_{i j}\right.$ is the connection weight between the nodes $i$ and $j$ in the network and $\theta_{j}$ is the threshold of the $j$ node);

Step 2: Read the pre-processed input vector $X_{j}$ and the desired output vector $Y_{j}$;

Step 3: Calculate the actual output $O: o_{j}=f\left(\sum w_{i j} x_{i}\right)$. Where sigmoid function is $f(x)=\frac{1}{1+e^{-(x-\theta)}}$;

Step 4: Correct the weights, back propagation;

Weight correction: $W_{i j}(t+1)=\alpha \delta_{i} O_{i}+W_{i j}(t)$, Threshold Correction: $\theta_{j}(t+1)=\theta_{j}(t)+\beta \delta_{i}$, in which $\alpha$ is the learning factor, $\beta$ is the momentum factor that accelerates the convergence.

Step 5: Calculate the error. $E=\frac{1}{2} \sum_{j}\left(o_{j}-y_{j}\right)^{2}$;

Repeat the above steps until the error meets the requirements.

\subsection{Improved gray BP neural network model}

\subsubsection{Set up the BP network model of residual series}

Assume the residual of the time series $X^{(0)}=\left\{x^{(0)}(1), x^{(0)}(2), \cdots, x^{(0)}(n)\right\}$ and the predicted value $\left\{\hat{x}^{(0)}(k)\right\} k=1,2, \cdots n$ using the $\operatorname{GM}(1,1)$ model is denoted as $e^{(0)}(k)$, scilicet $e^{(0)}(k)=x^{(0)}(k)-\hat{x}^{(0)}(k)$.

Assume $\left\{e^{(0)}(k)\right\} \quad k=1,2, \cdots n$ be the residual sequence; $S$ is the prediction order, it use the information of $e^{(0)}(k-1), e^{(0)}(k-2), \cdots, e^{(0)}(k-S)$ to predict the value of $k$ time. Then $e^{(0)}(k-1), e^{(0)}(k-2), \cdots, e^{(0)}(k-S)$ is regarded as the 
input sample of BP network and the value of $e^{(0)}(k)$ is taken as the expected value.

\subsubsection{Determine the predicted value of $\left\{\hat{e}^{(0)}(k)\right\}$}

The BP network is used to predict residual sequence $\left\{e^{(0)}(k)\right\}$, and the predicted value is $\left\{\hat{e}^{(0)}(k)\right\} k=1,2 \cdots n$. On this basis, calculate the new predictive value:

$$
\hat{x}^{(0)}(k, 1)=\hat{x}^{(0)}(k)+\hat{e}^{(0)}(k), \quad(k=1,2, \cdots n)
$$

$\hat{x}^{(0)}(k, 1)$ is the gray BP neural network combination model prediction results.

\section{Prediction of Coal Demand in Henan Province}

\subsection{Data Sources:}

The historical data of total coal consumption from 2009 to 2016 in Henan Province (data from Henan Statistical Yearbook 2009-2016) are shown in Table 1.

Tab.1 The original sample data

\begin{tabular}{|c|c|c|c|}
\hline years & $\begin{array}{c}\text { coal Consumption } \\
\text { (Ten thousand } \\
\text { tons of standard } \\
\text { coal) }\end{array}$ & years & $\begin{array}{c}\text { coal Consumption } \\
\text { (Ten thousand } \\
\text { tons of standard } \\
\text { coal) }\end{array}$ \\
\hline 2009 & 11868 & 2013 & 16547 \\
\hline 2010 & 12753 & 2014 & 17183 \\
\hline 2011 & 14188 & 2015 & 18072 \\
\hline 2012 & 15643 & 2016 & 19256 \\
\hline
\end{tabular}

\subsection{Forecast of Coal Demand in Henan}

First of all, we model the data in Table 1, the time response is:

$$
\hat{x}^{(1)}(k+1)=206732797 e^{0.0626 k}-194864.797
$$

The fitting results from 2002 to 2011 are shown in Table 2.

Tab.2 the fitted values and residual error based on GM $(1,1)$

\begin{tabular}{|c|c|c|c|c|}
\hline years & $\begin{array}{c}\text { Actual value } \\
x^{(0)}(k)\end{array}$ & $\begin{array}{c}\text { Fit values } \\
\hat{x}^{(0)}(k)\end{array}$ & $\begin{array}{c}\text { The Residual } \\
e^{(0)}(k)\end{array}$ & $\begin{array}{c}\text { Relative } \\
\text { error } \\
(\%)\end{array}$ \\
\hline 2009 & 11868 & 11868 & 0 & 0 \\
\hline 2010 & 12753 & 13350 & 597 & 4.68 \\
\hline 2011 & 14188 & 14212.1 & 23.1 & 0.16 \\
\hline 2012 & 15644 & 15129.9 & -515.1 & 3.28 \\
\hline
\end{tabular}

\begin{tabular}{|l|c|c|c|c|}
\hline 2013 & 16547 & 16106.9 & -440.1 & 2.66 \\
\hline 2014 & 17183 & 17147.1 & -35.9 & 2.09 \\
\hline 2015 & 18072 & 18254.4 & 182.4 & 1.01 \\
\hline 2016 & 19256 & 19433.2 & 177.2 & 0.92 \\
\hline
\end{tabular}

Secondly, the BP network model is established for the value of residual sequence in the table 2, and then modifies the fitting value. This paper adopts three-layer BP neural network structure. The data of the residuals of the previous three years $(\mathrm{S}=3)$ are selected as the input samples of the sequence, and the data of the next year are outputted as the network.

The network parameters are as follows: (1) The number of input nodes is 3 , the number of hidden nodes is 10 and the number of output nodes is 1. (2) The training function is trainglm, the hidden layer transfer function is logsig function, the output layer (3) the initial learning factor is 0.05 ; (4) allow the error to take 0.001 ;

At the same time, in order to improve the network training rate, the nonlinear least squares (L-M) optimization algorithm is introduced in the BP algorithm. From 2012 to 2016, the coal consumption of china was trained by gray BP neural network model is shown in Table 3

Tab.3 Fitted result and errors using Grey BP Neural Network model

\begin{tabular}{|c|c|c|c|c|}
\hline years & $\begin{array}{c}\text { Actual } \\
\text { value } \\
x^{(0)}(k)\end{array}$ & $\begin{array}{c}\text { Fit } \\
\text { values } \\
\hat{x}^{(0)}(k)\end{array}$ & $\begin{array}{c}\text { The } \\
\text { Residual } \\
e^{(0)}(k)\end{array}$ & $\begin{array}{c}\text { Relative } \\
\text { error } \\
(\%)\end{array}$ \\
\hline 2012 & 15644 & 15803.5 & -159.6 & 1.02 \\
\hline 2013 & 16547 & 16699.3 & -152.2 & 0.92 \\
\hline 2014 & 17183 & 17185.1 & -1.7 & 0.01 \\
\hline 2015 & 18072 & 17999.9 & 72.3 & 0.41 \\
\hline 2016 & 19256 & 19198.2 & 57.8 & 0.31 \\
\hline
\end{tabular}

From Table 3, it can be seen that the model has high prediction accuracy. Using the combined forecasting model, we can forecast the coal demand in China from 2017 to 2020, and the results are shown in Table 4 .

Tab.4 Predicted results of coal demand from 2017 to 2020 in Henan

\begin{tabular}{|c|c|c|c|c|}
\hline years & 2017 & 2018 & 2019 & 2020 \\
\hline $\begin{array}{c}\text { Predictive } \\
\text { value } \\
\text { (Ten } \\
\text { thousand tons } \\
\text { of standard } \\
\text { coal) }\end{array}$ & 20688.1 & 22024 & 23446.3 & 24960.4 \\
\hline
\end{tabular}




\section{Conclusions}

In order to improve the prediction accuracy, this paper constructs a combined forecasting model of gray BP artificial neural network to fit and predict the total coal consumption in Henan Province. The results show that the model has both high fitting accuracy and high prediction Accuracy. The forecast results show that the demand for coal in Henan will maintain a rapid growth trend, which is basically in line with the actual situation of the Central Plains Economic Zone and the economic development in Henan Province. The predicted data have certain reference value.

\section{References}

1. Liu Si-feng, Xie Nai-ming, etc . Gray system theory and its application [M] Beijing: Science Press, 2008: $155 \sim 157$.

2. Zhu Kai, Wang Zheng-lin. Proficient in MATLAB neural network $[\mathrm{M}]$. Beijing: Electronic Industry Press, 2015: $193 \sim 198$.

3. Fan Iv-an, Pan Zhong-qiang, et al. Application of gray GM $(1,1)$ model in coal demand forecast of Henan Province [J] .Journal of Coal Technology, 2016 (10): 7-9.

4. Wang Qiu-ming, Liu Ke-cheng, Gao Hui-ying. Process quality prediction based on gray theory and BP neural network [J]. Journal of Beijing Institute of Technology, 2016,3 1 (2): 249 -252.
5. Cao Lei, Zhong Fei, Zhang Ming-ji. Construction scale of urban construction land in Chongqing based on gray BP neural network [J] .Journal of Jiangxi Agricultural University, 2017,23 (8): 188 - 189.

6. GAO Xi-jing, WANG Lai-bin et al . Prediction of gas content in coal seams based on gray relational grade BP neural network [J] .Journal of Coal Technology, 2017 (8): 16-19.

7. WANG Li-jie, SUN Ji-hu. Prediction Model of Coal Demand Based on Gray System Theory [J] .Journal of Coal Research, 2016, (6): 333 - 336. 